\title{
OPEN Exploring genetic alterations in circulating tumor DNA from cerebrospinal fluid of pediatric medulloblastoma
}

\author{
Yanling Sun ${ }^{1}$, Miao Li ${ }^{1}$, Siqi Ren ${ }^{1}$, Yan Liu ${ }^{1}$, Jin Zhang ${ }^{1}$, Shuting $\mathrm{Li}^{1}$, Wenchao Gao ${ }^{1}$, \\ Xiaojun Gong ${ }^{1}$, Jingjing Liu ${ }^{1}$, Yuan Wang ${ }^{1}$, Shuxu Du ${ }^{1}$, Liming Sun ${ }^{1}$, Wanshui Wu ${ }^{1 凶} \&$ \\ Yongji Tian ${ }^{2 凶}$
}

Medulloblastoma (MB) is the most common type of brain malignancy in children. Molecular profiling has become an important component to select patients for therapeutic approaches, allowing for personalized therapy. In this study, we successfully identified detectable levels of tumor-derived cellfree DNA (cfDNA) in cerebrospinal fluid (CSF) samples of patients with MB. Furthermore, cfDNA from CSF can interrogate for tumor-associated molecular clues. MB-associated alterations from CSF, tumor, and post-chemotherapy plasma were compared by deep sequencing on next-generation sequencing platform. Shared alterations exist between CSF and matched tumor tissues. More alternations were detected in circulating tumor DNA from CSF than those in genomic DNA from primary tumor. It was feasible to detect MB-associated mutations in plasma of patients treated with chemotherapy. Collectively, CSF supernatant can be used to monitor genomic alterations, as a superior technique as long as tumor-derived cfDNA can be isolated from CSF successfully.

Medulloblastoma (MB) is the most common primary malignant tumor in children, accounting for $20 \%$ of all pediatric intracranial tumors ${ }^{1}$. Currently, MB is recognized as an umbrella term that encompasses various molecular pathological entities. Based on 2016 World Health Organization (WHO) classification of central nervous system (CNS) tumors, MB was divided into four subgroups, i.e., WNT, SHH, Group 3 and Group $4^{2}$. About one-third of children with MB have cerebrospinal fluid, cranial or spinal metastases at diagnosis ${ }^{3}$. Patients who suffer from recurrence or tumor progression, even with advanced treatment protocols, have dismal prognosis. Nowadays, cancer genome landscape is essential for diagnosis, prognostic evaluation and treatment selection. Unfortunately, for patients with tumor progression or recurrence, it is difficult to detect molecular alterations, because of unobtainable tumor tissue and lack of biopsy sample. Only advanced imaging technologies, such as MRI, CT and PET, are applied to diagnose and monitor progressive disease for MB.

The next-generation sequencing (NGS) has been applied to sequence circulating tumor DNA (ctDNA) from blood, especially for identifying EGFR mutations in non-small cell lung cancer. However, blood-brain barrier impedes detection of ctDNA in blood of a patient with brain tumor ${ }^{4}$. Recently, accumulating evidence has supported that circulating cell-free nucleic acids including DNA (cfDNA) and RNA (cfRNA), and even proteins in cerebrospinal fluid (CSF) are potential to revolutionize diagnosis and clinical care for CNS tumors, especially for glioblastoma (GBM) and $\mathrm{MB}^{5}$. We aim to explore the feasibility of liquid biopsy using CSF to facilitate diagnosis and to predict prognosis in $\mathrm{MB}$.

To decipher pathogenesis of $\mathrm{MB}$ and apply targeted therapy, it is necessary to explore genetic background in different types of samples, such as tumor tissue and CSF, based on NGS platform. Because in our previous clinical genetic test reports, few MB-associated alterations were detected in tumor tissue. Therefore, deep sequencing on ctDNA in CSF and formalin-fixed paraffin-embedded (FFPE) tissue was performed to obtain comprehensive genome profiling. We hypothesized that genetic alteration in CSF may serve as a complementary role to tissue for monitoring disease progression.

\footnotetext{
${ }^{1}$ Department of Pediatrics, Beijing Shijitan Hospital, Capital Medical University, 10E. Tieyi Road, Yangfangdian, Haidian District, Beijing, China. ${ }^{2}$ Department of Pediatric Neurosurgery, Beijing Tiantan Hospital, Capital Medical University, 119E. South Fourth Ring West Road, Fengtai District, Beijing, China. ${ }^{\square}$ email: wuwanshui2018@ ccmu.edu.cn; tyjpumc@163.com
} 
Circulating cell-free DNA (cfDNA) is present in body fluid of healthy individuals as well as patients with cancer, such as plasma, urine and CSF. Circulating tumor DNA (ctDNA) is thought to be shed into circulation by apoptotic and necrotic tumor cells in patients with cancer ${ }^{6}$. Thus detection of ctDNA is of great value for early diagnosis of malignancy. For detecting ctDNA in the blood, the content of ctDNA is lower at earlier stage, compared to relatively late-stage ${ }^{4,7}$. Additionally, the difference of fragments size in ctDNA and cfDNA was utilized for early diagnosis of malignancies ${ }^{8}$. Furthermore, high-grade (WHO grades III and IV) brain tumors were more likely to harbor detectable ctDNA in CSF than low-grade ones ${ }^{9}$. Therefore, ctDNA detection in CSF plays a predictive role in monitoring disease progression.

Here, we determined the presence or absence of cfDNA in 58 CSF samples. In order to decipher medulloblastoma-associated alterations, deep sequencing based on NGS platform was applied to examine CSF, tumor tissue and blood in patients with MB. Notably, positive cfDNA in CSF is associated with disease progression or metastasis. It was more likely to detect medulloblastoma-associated alterations in CSF rather than tumor tissue. Shared alterations between CSF and matched tumor tissues could be detected when sample collected in short time interval.

\section{Methods}

Patients and sample collection. The protocol was approved by the institutional review board of Research Ethics Committee at Beijing Shijitan Hospital. Written informed consent was obtained from their parents of MB patients. All experiments were carried out in accordance with relevant guidelines and regulations.

In this prospective study, 58 patients with MB were recruited from Beijing Tiantan Hospital of Capital Medical University and Beijing Shijitan Hospital of Capital Medical University between April 2019 and Dec 2019. For each patient, CSF was collected by lumbar puncture or at the time of surgery. Additionally, tumor tissue samples were collected from 4 patients on primary-care and 7 patients with relapse. The matched plasma was isolated from 5 patients with relapse and treated with adjuvant radiotherapy or chemotherapy. Furthermore, whole blood was obtained from each patient, serving as germline DNA control.

Isolation of cfDNA from CSF and plasma. The median volume of collected CSF is $5 \mathrm{~mL}$. Fresh CSF was stored at $4{ }^{\circ} \mathrm{C}$, and centrifugated (at $4{ }^{\circ} \mathrm{C}, 1400 \mathrm{rpm}$, for $5 \mathrm{~min}$ ) within $2-3 \mathrm{~h}$ after collection. Cellular pellet was discarded. The sterile centrifuge tube was pre-cooled at $-20^{\circ} \mathrm{C}$. CSF supernatant was transferred to a cryotubes and stored at $-80^{\circ} \mathrm{C}$. Immediately before use, CSF was thawed in a thermostat water bath at $37^{\circ} \mathrm{C}$. Then, cfDNA was extracted from CSF supernatant or plasma according to the manufacturer's protocol using QIAamp Circulating Nucleic Acid Kit (catalog \#55114; QIAGEN, Valencia, CA).

Isolation of tumor/germline DNA. Isolation of genomic DNA from FFPE tumor tissue or matched whole blood leukocytes was performed using QIAamp DNA FFPE Tissue Kit (Qiagen, Hilden, Germany) and blood/cell culture DNA kit (Qiagen, Valencia, CA, USA), respectively. Concentration of isolated (cell-free and genomic) DNA was quantified with Qubit 2.0 Fluorometer using Qubit dsDNA HS Assay kit (Life Technologies, Carlsbad, California, US) according to the manufacture's protocol. DNA was stored at $-20^{\circ} \mathrm{C}$ until it was used.

Analysis of cfDNA and ctDNA. The presence and absence of cfDNA was inferred according to isolated DNA fragment length distribution, instead of quantity. To verify isolated cfDNA, the size of cfDNA fragment was assessed using Agilent 2100 Bioanalyzer. If peak appeared at 150-200 base pair (bp), it is considered as cfDNA, otherwise (> $1500 \mathrm{bp}$ ) as CSF cellular genomic DNA. And NGS library construction was not performed against CSF cellular genomic DNA. The detection method cannot determine whether cfDNA is derived from tumor tissue. Therefore, we adopted the term "cfDNA". The term "ctDNA" was used only when tumor-associated mutations were detected in CSF or plasma ${ }^{10}$.

DNA library construction and NGS sequencing. First, tumor genomic DNA was fragmented into 150-200 bp fragments with Bioruptor Pico. Libraries of fragmented DNA and cfDNA were constructed with KAPA Library Amplification Kit (KK2611/KK2612) according to standard protocols. Next, cfDNA libraries were captured with a designed 500-gene or 952-gene panel (Agilent), which contained the majority of brain tumor related genes. The genomic DNA libraries were applied with whole exome sequencing (WES). The captured samples were subjected to paired-end sequencing on the Illumina NovaSeq 6000 platform. Finally, the low quality reads were removed based on bioinformatics; while the high quality reads were mapped on human reference sequence (hg19) (Human Genome version 19) using tool Burrows-Wheeler Aligner software (BWA) ${ }^{11}$. The SNVs and In/Dels were detected using Genome Analysis Toolkit (GATK) ${ }^{12}$ and VarScan $2^{13}$ and filtered out by dbSNP and 1000 Genome datasets. We selected at least 3 mutated reads with quality scores of $>30$. In this study, we focused on mutations located in exons, such as nonsynonymous, synonymous, frameshift, non-frameshift and splicing sites. All the genes tested were verified with Integrative Genomics Viewer (IGV).

Statistical analysis. Associations between CSF-cfDNA and clinical characteristics including age and quantity of DNA in CSF were evaluated by Mann-Whitney U tests. Clinical features such as recurrence, metastasis, tumor progression and quantity of DNA in CSF were compared between positive and negative CSF-cfDNA groups with Fisher's exact tests or chi-square tests. All statistical tests were two-sided with a $p \leq 0.05$ defined as statistical significance. 


\section{Results}

The basic characteristics of patients. The patient population ( $64 \%$ male and $36 \%$ female) included infants, children or teenager, aged at 2-15 years old (median: 7). Demographic and clinical features were summarized in Table 1, including age, gender, medial histology, primary tumor location, initial diagnosis ( $\mathrm{M}$ stage, molecular subgroup), recurrence, progression, with or without detectable CSF-cfDNA, and quantity of DNA in CSF. Tumors were located in the 4 th ventricle in the majority of patients $(n=48) .7$ patients had spinal spread, 3 had spinal solitary metastasis, 2 had craniospinal (cerebrospinal; cerebellum and spinal axis) spread and one had subventricular spread. Other tumors were located in vermis $(n=5)$, cerebellar hemisphere $(n=4)$ or cerebellopontine angle (CPA) $(n=1)$. Totally, 15 patients developed distant metastasis or local spread. Disease recurrence occurred in 7 patients. Among 58 patients, 15 had detectable cfDNA in CSF (as analyzed by Agilent 2100 Bioanalyzer). Eight patients developed progressive disease (PD) or died of PD. One patient exhibited partial remission $(\mathrm{PR})$ after chemotherapy and surgery. The rest of patients achieved stable disease $(\mathrm{SD})(\mathrm{n}=3)$ or complete remission (CR) $(\mathrm{n}=46)$ after surgery, nevertheless, the patient 192D0463 died from radiation (RT) complication.

Relationship between CSF-cfDNA and clinical characteristics. In our patients with MB, CSFcfDNA was not associated with age ( $p=0.50$, Mann-Whitney $\mathrm{U}$ test $)$ or gender $(p=0.37$, chi-square test). However, the presence or absence of cfDNA in CSF was associated with several clinical features, including recurrence $(p=0.0098$, Fisher's exact test), metastasis $(p=0.001$, Fisher's exact test), tumor progression $(p=0.003$, Fisher's exact test), as well as quantity of DNA in CSF ( $p<0.001$, Mann-Whitney U test) (Table 2$)$. The median quantity of DNA in CSF with cfDNA (279 ng) is higher than that without cfDNA (11 ng).

Medulloblastoma-associated alteration detected in CSF, tumor and plasma. For this study, eleven patients with MB were selected for genomic analysis. Seven patients developed recurrent diseases. Tissue DNA, CSF or plasma cfDNA from 6 patients were subjected to sequencing on NGS platform. The isolated tumor and germline DNA samples were sequenced with WES. The cfDNA isolation yielded from CSF and plasma was sequenced on NGS platforms with 500-gene panel, except for 192D0360 with 952-gene panel. Because of cancer genes were detected in those patients' CSF or plasma, the term "cfDNA" be called "ctDNA" in following text ${ }^{10}$. Medulloblastoma-associated alterations detected in ctDNA from CSF were more abundantly present than genomic DNA from tumor tissue (Fig. 1). The most common genetic alterations were KMT2D (32.0\%), KMT2C (28.0\%), SMARCA4 (24.0\%), BCOR (20.0\%), TP53 (12.0\%), PTCH1 (8\%), EP300 (8\%), NF1 (8\%), SETD2 (8\%), $M E D 12(8 \%)$ and SPEN (8\%). Notably, BCOR alterations were the most frequent in ctDNA from plasma. The presence of medulloblastoma-associated mutations in plasma may be due to the fact that patients with recurrent disease had been treated with adjuvant radiotherapy or chemotherapy. It was indicated that blood-brain barrier system could be damaged or destroyed in response to radiotherapy or chemotherapy ${ }^{14-17}$. It is surprising that medulloblastoma-associated somatic mutations from tumor tissues were detected in only $36 \%$ (4/11) of patients. We identified nonsynonymous SNVs in tumor tissues, such as SMARCA4 (in 192D0360T and 192D0641T), $B C O R$ and $L R P 1 B$ in 192D0641T, as well as frameshift mutations of KMT2D in 192D0695T and truncating mutations of PTCH1 in 192D0583T. Although it is possible that the low concordance between samples from the same individual could be resulted from the number of different detected alterations, the common medulloblastoma-associated alterations in tumor tissue were few in samples with more genes detected, such as 192D0583T, 192D0629T and 192D0674T (Fig. 1 and Fig. S1). Additionally, shared alterations between tissue and CSF or plasma were only found in case 192D0360, 192 D0695 and 192D0641 (Fig. S1). It was not an obvious trend indicating that the increasing concordance of alterations between samples changed along with improvement of detectable genes.

Shared genetic alterations between ctDNA from CSF and genomic DNA from tumor tissue. NGS-based detection of alterations was performed against nine paired tumor DNA and CSF-ctDNA to explore shared genetic mutations between CSF and tumor tissue. As shown in Table 3, shared alterations were detected in 2 out of 9 patients (22\%). Two nonsynonymous SNVs in SMARCA4 and SETD8 genes and 2 synonymous SNVs in DROSHA and MLLT3 genes were identified in case 192D0360. Frameshift mutation in KMT2D and nonsynonymous SNVs in SNCAIP were identified in case 192D0695. The most common alterations included truncating mutations in KMT2D ${ }^{18}$ and SNCAIP tandem duplication (somatic mutation), excluding from dominated somatic copy number aberrations (SCNA) in MB Group $4^{19}$. Nevertheless, no shared alterations were found in the remaining seven patients $(78 \%)$.

To clarify the reason about the detectable shared mutations, we then collected clinical information of the above nine patients (Table 4). We found that CSF collected by lumber puncture was only one month after operation for the case 192D0360 and 192D0593. The CSF from the case 192D0695 and 192D0640 was collected during operation. Shared alterations were found only in the case 192D0360 and 192D0695. The remaining CSF samples were collected at the time of recurrence, and mean time interval from the initial surgery is 15.7 months (range, 5.5-23 months). No shared alterations could be detected in those cases (Table 4). We guess that shared gene alterations might be detected only when the collecting time interval between CSF and matching tumor tissue was close.

\section{Discussion}

It is challenging to monitor disease progression for patients with CNS tumors since repeated surgical resection is unfeasible, while the pathology diagnosis that distinguish proliferative/aggressive tumor, pseudoprogression or necrosis directly influenced treatment decision-making process ${ }^{20}$. Therefore, detection of tumor-derived 


\begin{tabular}{|c|c|c|c|c|c|c|c|c|c|c|}
\hline Sample & Age (y) & Sex & Primary location & Histology & M stage & Molecular subgroup & Recurrence & CSF-cfDNA & DNA in CSF (ng) & Tumor progression \\
\hline 192D0695 & 5 & $\mathrm{M}$ & 4th ventricle & DMB & M0 & SHH & No & Positive & 14.7 & $\mathrm{CR}$ \\
\hline 192D0640 & 7 & $\mathrm{M}$ & 4th ventricle & CMB & M0 & Group 4 & No & Positive & 1995 & CR \\
\hline 192D0394 & 6 & M & 4th ventricle & DMB & M0 & SHH & No & Positive & 17.99 & CR \\
\hline 192D0360 & 3 & $F$ & $\begin{array}{l}\text { 4th ventricle and } \\
\text { spinal spread }\end{array}$ & CMB & M3 & Group4 & No & Positive & 84.8 & PD, die \\
\hline 192D0593 & 7 & M & $\begin{array}{l}\text { 4th ventricle and } \\
\text { spinal spread }\end{array}$ & CMB & M3 & Group 4 & No & Positive & 17.29 & SD \\
\hline 192D0630 & 6 & M & $\begin{array}{l}\text { 4th ventricle and } \\
\text { spinal spread }\end{array}$ & CMB & M3 & Group4 & No & Positive & 12.67 & PR \\
\hline 192D0479 & 9 & M & $\begin{array}{l}\text { 4th ventricle with spi- } \\
\text { nal solitary metastasis }\end{array}$ & CMB & M3 & Group4 & No & Positive & 12.88 & $\mathrm{CR}$ \\
\hline 202D0009 & 3 & M & $\begin{array}{l}\text { 4th ventricle and } \\
\text { spinal spread }\end{array}$ & СMB & M3 & Group 4 & No & Positive & 784 & Die \\
\hline 202D0037 & 7 & M & 4th ventricle & CMB & M1 & Group 4 & No & Positive & 90.4 & CR \\
\hline 192D0406 & 8 & $\mathrm{~F}$ & Cerebellar hemisphere & CMB & M1 & Group 3 & No & Positive & 28 & CR \\
\hline 192D0582 & 5 & F & Vermis & DMB & M0 & SHH & Yes & Positive & 24.22 & $\mathrm{PD}$ \\
\hline 192D0584 & 3 & M & $\begin{array}{l}\text { 4th ventricle with } \\
\text { craniospinal spread }\end{array}$ & LMB & M3 & SHH/Group 4 & Yes & Positive & 106.4 & PD, die \\
\hline 192D0629 & 5 & $\mathrm{M}$ & 4th ventricle & CMB & M0 & 1 & Yes & Positive & 173.6 & PD \\
\hline 192D0653 & 9 & $\mathrm{~F}$ & 4th ventricle & CMB & M0 & Group 4 & Yes & Positive & 777 & PD, die \\
\hline 192D0674 & 10 & M & $\begin{array}{l}\text { 4th ventricle and } \\
\text { spinal spread }\end{array}$ & CMB & M3 & Group 4 & Yes & Positive & 40.25 & SD \\
\hline 192D0583 & 2 & $\mathrm{M}$ & Cerebellar hemisphere & CMB & M0 & SHH & Yes & Not detected & 11.13 & CR \\
\hline 192D0641 & 13 & $\mathrm{~F}$ & 4th ventricle & CMB & M0 & SHH & Yes & Not detected & 28.42 & PD \\
\hline 192D0663 & 2 & M & 4th ventricle & CMB & M0 & Group 3 & No & Not detected & 9.8 & PD \\
\hline 192D0463 & 6 & M & 4th ventricle & СMB & M0 & Group 4 & No & Not detected & 6.8 & $\begin{array}{l}\text { Die from RT compli- } \\
\text { cation }\end{array}$ \\
\hline 202D0024 & 7 & M & $\begin{array}{l}\text { 4th ventricle with spi- } \\
\text { nal solitary metastasis }\end{array}$ & MEBN & M3 & SHH & No & Not detected & 5.76 & SD \\
\hline 192D0408 & 12 & M & $\begin{array}{l}\text { 4th ventricle and } \\
\text { spinal spread }\end{array}$ & DMB & M3 & SHH & No & Not detected & 30.03 & $\mathrm{CR}$ \\
\hline 202D0005 & 7 & M & $\begin{array}{l}\text { 4th ventricle with spi- } \\
\text { nal solitary metastasis }\end{array}$ & DMB & M3 & SHH & No & Not detected & 8.12 & CR \\
\hline 192D0331 & 7 & F & $\begin{array}{l}\text { 4th ventricle and sub- } \\
\text { ventricular spread }\end{array}$ & DMB & M2 & SHH & No & Not detected & 5.48 & CR \\
\hline 192D0604 & 2 & F & $\begin{array}{l}\text { 4th ventricle with } \\
\text { craniospinal spread }\end{array}$ & CMB & M3 & Group 4 & No & Not detected & 9.8 & CR \\
\hline 192D0669 & 8 & M & $\begin{array}{l}\text { 4th ventricle and } \\
\text { spinal spread }\end{array}$ & CMB & M3 & Group 4 & No & Not detected & 7.42 & $\mathrm{CR}$ \\
\hline 192D0657 & 11 & M & 4th ventricle & DMB & M0 & SHH & No & Not detected & 77 & CR \\
\hline 192D0362 & 5 & $\mathrm{~F}$ & 4th ventricle & DMB & M0 & WNT & No & Not detected & 9.92 & CR \\
\hline 192D0448 & 5 & $\mathrm{M}$ & 4th ventricle & CMB & M0 & Group4 & No & Not detected & 6.88 & $\mathrm{CR}$ \\
\hline 192D0449 & 5 & M & 4th ventricle & DMB & M0 & Group3 & No & Not detected & 9.59 & CR \\
\hline 192D0480 & 9 & $\mathrm{M}$ & 4th ventricle & CMB & M0 & WNT & No & Not detected & 8.4 & CR \\
\hline 192D0532 & 11 & M & 4th ventricle & CMB & M0 & Group4 & No & Not detected & 8.2 & CR \\
\hline 192D0542 & 8 & $\mathrm{~F}$ & 4th ventricle & CMB & M0 & WNT & No & Not detected & 7.6 & $\mathrm{CR}$ \\
\hline 192D0591 & 13 & M & 4th ventricle & CMB & M0 & Group4 & No & Not detected & 12.74 & CR \\
\hline 192D0627 & 6 & $\mathrm{~F}$ & 4th ventricle & CMB & M0 & WNT & No & Not detected & 8.4 & CR \\
\hline 192D0571 & 4 & $\mathrm{M}$ & 4th ventricle & CMB & M0 & Group3 & No & Not detected & 7.63 & CR \\
\hline 192D0574 & 5 & M & 4th ventricle & CMB & M0 & Group4 & No & Not detected & 12.39 & CR \\
\hline 192D0585 & 8 & $\mathrm{~F}$ & 4th ventricle & CMB & M0 & WNT & No & Not detected & 7.91 & CR \\
\hline 192D0594 & 8 & $\mathrm{M}$ & 4th ventricle & CMB & M0 & Group4 & No & Not detected & 12.95 & CR \\
\hline 192D0631 & 3 & $\mathrm{M}$ & 4th ventricle & DMB & M0 & Group4 & No & Not detected & 5.39 & CR \\
\hline 192D0646 & 8 & $\mathrm{~F}$ & 4th ventricle & CMB & M0 & Group4 & No & Not detected & 8.26 & CR \\
\hline 192D0652 & 15 & $\mathrm{M}$ & 4th ventricle & DMB & M0 & SHH & No & Not detected & 3.78 & CR \\
\hline 192D0655 & 8 & $\mathrm{M}$ & 4th ventricle & CMB & M0 & 1 & No & Not detected & 8.19 & $\mathrm{CR}$ \\
\hline 192D0662 & 2 & $\mathrm{~F}$ & 4th ventricle & CMB & M0 & Group3 & No & Not detected & 6.3 & CR \\
\hline 192D0671 & 5 & $\mathrm{M}$ & 4th ventricle & DMB & M0 & SHH & No & Not detected & 3.5 & CR \\
\hline 192D0696 & 2 & $\mathrm{M}$ & 4th ventricle & CMB & M0 & Group4 & No & Not detected & 11.9 & CR \\
\hline 202D0004 & 6 & $\mathrm{~F}$ & 4th ventricle & DMB & M0 & Group3 & No & Not detected & 8.54 & CR \\
\hline 202D0007 & 11 & F & 4th ventricle & CMB & M0 & WNT & No & Not detected & 15.12 & CR \\
\hline \multicolumn{11}{|l|}{ Continued } \\
\hline
\end{tabular}




\begin{tabular}{|c|c|c|c|c|c|c|c|c|c|c|}
\hline Sample & Age (y) & Sex & Primary location & Histology & M stage & Molecular subgroup & Recurrence & CSF-cfDNA & DNA in CSF (ng) & Tumor progression \\
\hline 202D0013 & 2 & $\mathrm{~F}$ & 4th ventricle & DMB & M0 & SHH & No & Not detected & 3.71 & $\mathrm{CR}$ \\
\hline 192D0590 & 8 & M & 4th ventricle & $\mathrm{DMB}$ & M0 & SHH & No & Not detected & 8.05 & $\mathrm{CR}$ \\
\hline $202 \mathrm{D} 0020$ & 8 & M & 4 th ventricle & CMB & M0 & Group4 & No & Not detected & 6.3 & $\mathrm{CR}$ \\
\hline $202 \mathrm{D} 0029$ & 7 & $\mathrm{~F}$ & 4th ventricle & CMB & M0 & WNT & No & Not detected & 6.48 & $\mathrm{CR}$ \\
\hline 192D0407 & 3 & $\mathrm{~F}$ & $\mathrm{CPA}$ & DMB & M0 & $\mathrm{SHH}$ & No & Not detected & 7.49 & $\mathrm{CR}$ \\
\hline 192D0361 & 6 & $\mathrm{M}$ & Cerebellar hemisphere & CMB & M0 & SHH & No & Not detected & 6.02 & CR \\
\hline $192 \mathrm{D} 0425$ & 5 & $\mathrm{M}$ & Cerebellar hemisphere & L/AMB & M0 & $\mathrm{SHH}$ & No & Not detected & 6.51 & $\mathrm{CR}$ \\
\hline 192D0484 & 8 & $\mathrm{~F}$ & Vermis & $\mathrm{CMB}$ & M0 & WNT & No & Not detected & 10.16 & CR \\
\hline 192D0592 & 12 & $\mathrm{~F}$ & Vermis & $\mathrm{CMB}$ & M0 & Group4 & No & Not detected & 7.7 & CR \\
\hline 192D0626 & 9 & $\mathrm{M}$ & Vermis & $\mathrm{CMB}$ & M0 & Group4 & No & Not detected & 7.14 & $\mathrm{CR}$ \\
\hline 202D0032 & 8 & $\mathrm{~F}$ & Vermis & CMB & M0 & Group4 & No & Not detected & 6.08 & CR \\
\hline
\end{tabular}

Table 1. Clinical characteristics of 58 patients with medulloblastoma. CR, complete remission; PD, progressive disease; SD, stable disease; PR, partial remission; CPA, cerebellopontine angle.

\begin{tabular}{|c|c|c|c|c|}
\hline Characteristic & All patients $(n=58)$ & With cfDNA $(n=15)$ & Without cfDNA $(n=43)$ & $p$ value \\
\hline Age (y); median (range) & $7(2-15)$ & $6(3-10)$ & $7(2-15)$ & 0.50 \\
\hline \multicolumn{4}{|l|}{ Sex } & 0.37 \\
\hline Male; n (\%) & $37(64)$ & $11(73)$ & $26(60)$ & \\
\hline Female; n (\%) & $21(36)$ & $4(27)$ & $17(40)$ & \\
\hline \multicolumn{4}{|l|}{ Recurrent, n (\%) } & 0.0098 \\
\hline No & $51(88)$ & $10(67)$ & $41(95)$ & \\
\hline Yes & $7(12)$ & $5(33)$ & $2(5)$ & \\
\hline \multicolumn{4}{|l|}{ M stage, n (\%) } & 0.001 \\
\hline M0 & $43(74)$ & $6(40)$ & $37(86)$ & \\
\hline M1-3 & $15(26)$ & $9(60)$ & $6(14)$ & \\
\hline \multicolumn{4}{|l|}{ Tumor progression } & 0.003 \\
\hline CR or SD or PR & $50(86)$ & $9(60)$ & $41(95)$ & \\
\hline PD or Dead & $8(14)$ & $6(40)$ & $2(5)$ & \\
\hline \multicolumn{4}{|c|}{ Quantity of DNA in CSF (ng) } & $<0.001$ \\
\hline Median (range) & $80(4-1995)$ & $279(13-1995)$ & $11(4-77)$ & \\
\hline
\end{tabular}

Table 2. Associations between clinical characteristics and CSF-cfDNA.

cfDNA in CSF has become a minimally invasive approach to dynamically monitor tumor progression, which can facilitate targeted therapy for cancer patients.

In our study, cfDNA from 43 out of 58 CSF samples could not be detected. The presence of tumor-derived cfDNA from CSF was significantly related to primary location of tumor adjacent to CSF reservoir. The CNS neoplasms located in brain or spinal cord, where lesions adjacent to CSF reservoir were much more likely to harbor tumor-derived cfDNA in $\mathrm{CSF}^{9}$. In our patient population, tumor lesions interacted with CSF reservoir directly. The difference may be also caused by limitation of current detection techniques using CSF as liquid biopsy in childhood MB. Notably, CSF-cfDNA was undetected in the vast majority of patients who exhibited CR after surgery (without metastasis). cfDNA in CSF was detected in 15 patients. Among these 15 patients, five developed postoperative recurrence and $\mathrm{PD}$, and 7 with initial therapy had varying degrees of metastasis (M1 or M3) or PD and even died of rapid PD. Only 3 patients achieved CR (without metastasis) after surgery in several months (range from 2 to 6 months). Furthermore, clinical stage, such as recurrence, metastasis and PD between positive and negative CSF-cfDNA was different statistically. Similar to previous studies, the levels of tumor-derived cfDNA in CSF reflected response to therapy or $\mathrm{PD}^{21}$. Additionally, the quantity of plasma-ctDNA was correlated with tumor burden, as a predictor for recurrence and progression ${ }^{10}$.

In our study, more medulloblastoma-associated gene mutations were identified in CSF than in tumor tissue (Fig. 1). This difference may be caused by sampling bias inherent in traditional biopsy and tumor heterogeneity. However, more tumor-derived genetic alterations were identified from CSF than tumor tissue, as important factors for pathogenesis of MB. Medulloblastoma-associated alterations were not detected in the majority of FFPE tumor tissues (Fig. 1). Similarly, genetic alterations in CSF-ctDNA were unable to be consistently detected for supratentorial brain tumors ${ }^{22}$. From another perspective, the mean sequencing depth for liquid biopsy $(2000 \times)$ is higher than tumor tissue $(110 \times)$, so that more alterations may be captured. Therefore, more reasonable sequencing scheme should be designed to verify our findings. Additionally, medulloblastoma-associated alterations were detected in blood, possibly attributed to adjuvant radiotherapy or chemotherapy for patients with postoperative recurrence. We may infer that brain tumor-related gene mutations are detectable in the blood of patients with 


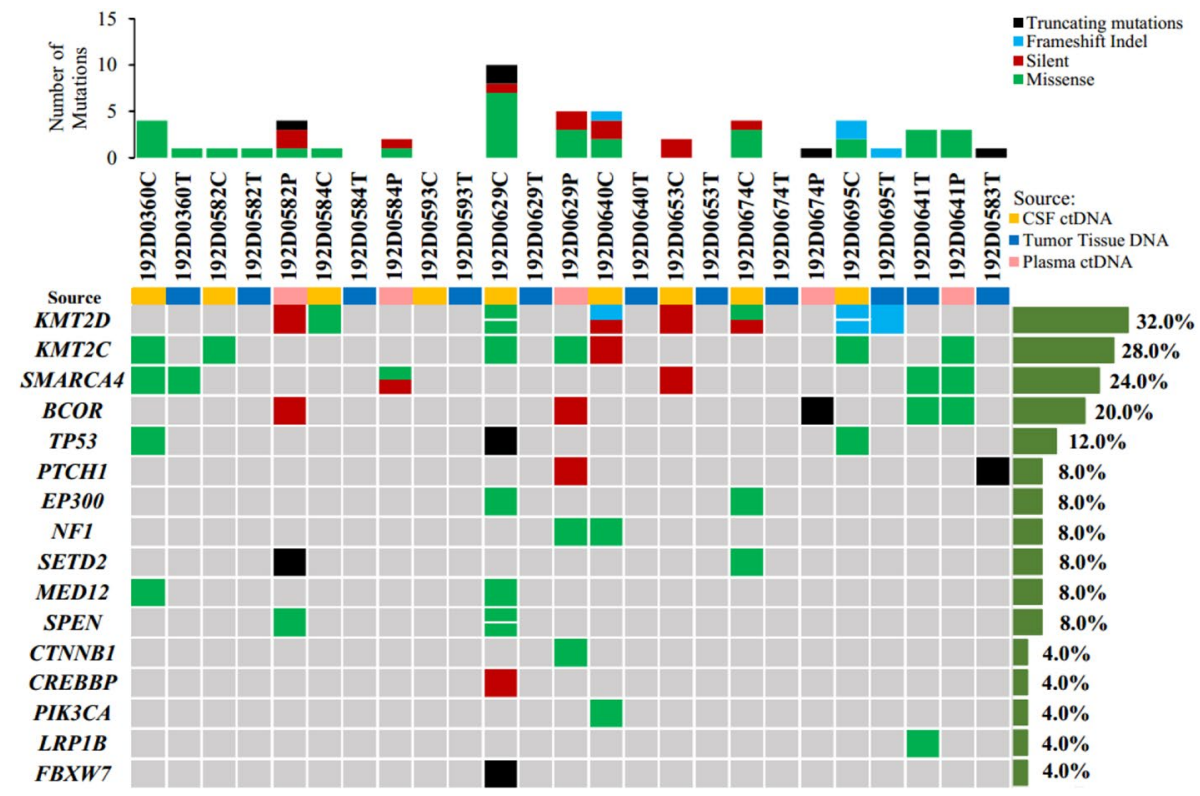

Figure 1. The genomic landscape of 11 medulloblastoma patients. Top, frequency of mutations per case; Bottom left, genetic alterations detected in 16 medulloblastoma-associated genes; Bottom right, frequency of mutations per gene in three different types of samples. C, CSF supernatant; T, tumor tissue; P, plasma.

\begin{tabular}{|c|c|c|c|c|c|c|c|c|}
\hline Sample & Chromosome & $\begin{array}{l}\text { Mutant } \\
\text { functional } \\
\text { type }\end{array}$ & Gene & Transcript & Location & cDNA Mutation & $\begin{array}{l}\text { AA } \\
\text { Mutation }\end{array}$ & MAF (\%) \\
\hline 192D0360C & chr5: 31409426 & $\begin{array}{l}\text { synony- } \\
\text { mous SNV }\end{array}$ & DROSHA & NM_013235 & exon31 & c. $3681 \mathrm{G}>\mathrm{A}$ & p.A1227A & 46.60 \\
\hline 192D0360T & chr5: 31409426 & \begin{tabular}{|l|} 
synony- \\
mous SNV
\end{tabular} & DROSHA & NM_013235 & exon31 & c. $3681 \mathrm{G}>\mathrm{A}$ & p.A1227A & 48.30 \\
\hline 192D0360C & chr9: 20414280 & $\begin{array}{l}\text { synony- } \\
\text { mous SNV }\end{array}$ & $M L L T 3$ & NM_004529 & exon5 & c. $564 \mathrm{C}>\mathrm{T}$ & p.S188S & 2.00 \\
\hline 192D0360T & chr9: 20414280 & $\begin{array}{l}\text { synony- } \\
\text { mous SNV }\end{array}$ & MLLT3 & NM_004529 & exon5 & c. $564 \mathrm{C}>\mathrm{T}$ & p.S188S & 3.80 \\
\hline 192D0360C & $\begin{array}{l}\text { chr19: } \\
11132513\end{array}$ & $\begin{array}{l}\text { nonsynony- } \\
\text { mous SNV }\end{array}$ & SMARCA4 & NM_003072 & exon 19 & c. $2729 \mathrm{C}>\mathrm{T}$ & p.T910M & 40.90 \\
\hline 192D0360T & $\begin{array}{l}\text { chr19: } \\
11132513\end{array}$ & $\begin{array}{l}\text { nonsynony- } \\
\text { mous SNV }\end{array}$ & SMARCA4 & NM_003072 & exon19 & c. $2729 \mathrm{C}>\mathrm{T}$ & p.T910M & 6.00 \\
\hline 192D0360C & $\begin{array}{l}\text { chr12: } \\
123875223\end{array}$ & $\begin{array}{l}\text { nonsynony- } \\
\text { mous SNV }\end{array}$ & SETD8 & NM_020382 & exon3 & c. $179 \mathrm{C}>\mathrm{T}$ & p.P60L & 2.30 \\
\hline 192D0360T & $\begin{array}{l}\text { chr12: } \\
123875223\end{array}$ & $\begin{array}{l}\text { nonsynony- } \\
\text { mous SNV }\end{array}$ & SETD8 & NM_020382 & exon3 & c. $179 \mathrm{C}>\mathrm{T}$ & p.P60L & 3.20 \\
\hline 192D0695C & $\begin{array}{l}\text { chr5: } \\
121786553\end{array}$ & $\begin{array}{l}\text { nonsynony- } \\
\text { mous SNV }\end{array}$ & SNCAIP & NM_005460 & exon 10 & c. $2011 \mathrm{~A}>\mathrm{T}$ & p.M671L & 4.80 \\
\hline 192D0695T & $\begin{array}{l}\text { chr5: } \\
121786553\end{array}$ & $\begin{array}{l}\text { nonsynony- } \\
\text { mous SNV }\end{array}$ & SNCAIP & NM_005460 & exon 10 & c. $2011 \mathrm{~A}>\mathrm{T}$ & p.M671L & 8.00 \\
\hline 192D0695C & $\begin{array}{l}\text { chr12: } \\
49427079\end{array}$ & $\begin{array}{l}\text { frameshift } \\
\text { insertion }\end{array}$ & $K M T 2 D$ & NM_003482 & exon39 & c.11408_11409insTGGG & p.G3803fs & 14.20 \\
\hline 192D0695T & $\begin{array}{l}\text { chr12: } \\
49427079\end{array}$ & $\begin{array}{l}\text { frameshift } \\
\text { insertion }\end{array}$ & $K M T 2 D$ & NM_003482 & exon39 & c.11408_11409insTGGG & p.G3803fs & 13.70 \\
\hline
\end{tabular}

Table 3. The shared alterations detected from CSF and tumor tissue in 9 cases. C, CSF supernatant; T, Tumor tissue; MAF, Mutant allele fraction; AA, Amino acid.

MB who have been treated with radiotherapy or chemotherapy. However, since we did not collect blood samples from patients who were not treated with chemoradiotherapy, it remained unclear if mutation detection in ctDNA from plasma is applicable to all kinds of treated patients with brain tumors.

No or few medulloblastoma-associated alterations were detected from tumor tissue. Shared alterations were identified between CSF and matched tumor tissue in only 2 out of 9 patients. Shared missense mutations may contribute to pathogenesis of MB. For example, SMARCA4 forms a protein complex with CTNNB1, which 


\begin{tabular}{|l|l|l|l|l|l|}
\hline Sample & Gender & Onset age & Treatment stage & $\begin{array}{l}\text { Collecting time interval between } \\
\text { CSF and tumor }\end{array}$ & $\begin{array}{l}\text { Shared alterations between CSF } \\
\text { and tissue }\end{array}$ \\
\hline 192D0629 & M & 5 & Recurrence after surgery & 29 months after operation & N \\
\hline 192D0674 & M & 10 & Recurrence after surgery & 23 months after operation & N \\
\hline 192D0584 & M & 3 & Recurrence after surgery & 12 months after operation & N \\
\hline 192D0582 & F & 5 & Recurrence after surgery & 9 months after operation & N \\
\hline 192D0653 & F & 9 & Recurrence after surgery & 5.5 months after operation & N \\
\hline 192D0593 & M & 7 & Initial therapy & 1 month after operation & N \\
\hline 192D0360 & F & 3 & Initial therapy & 1 month after operation & Y \\
\hline 192D0695 & M & 5 & Initial therapy & During operation & Y \\
\hline 192D0640 & M & 7 & Initial therapy & During operation & N \\
\hline
\end{tabular}

Table 4. The time interval for sample collection between CSF and tumor tissue. Y, with shared alterations; N, without shared alterations.

remodels chromatin and participates in WNT pathway ${ }^{23}$. SETD8 (also known as KMT5A) regulates TP53 activity and expression ${ }^{24}$. KMT2D (also known as MLL2) acts as a universal oncogenic driver to regulate epigenetic alterations. SNCAIP encodes synphilin-1, by binding to $\alpha$-synuclein, which promotes the formation of Lewy bodies in the brains of patients with Parkinson's disease ${ }^{25,26}$. Interestingly, in both patients, time interval for sample collection between CSF and tumor tissue was very short. CSF was collected one month after operation for 192D0360 whereas during operation for 192D0695. By contrast, CSF was collected during operation for 192D0640 whereas one month after surgery for 192D0593, but no shared alterations were detected from these 2 cases. CSF was obtained from the remaining 5 samples after disease recurrence. It may be easier to detect shared mutations between CSF and matched tumor tissue when time interval for sample collection is relatively short. CSF-ctDNA from patients with MB provides a comprehensive and genetically representative genome profiling only at the time of CSF collection. Finally, this result is consistent with previous findings that genomic evolution of brain tumors can change significantly over time $\mathrm{e}^{27-32}$.

In conclusion, detectable cfDNA in CSF may predict disease progression. Deep sequencing on CSF-ctDNA derived from patients with MB may obtain more tumor-specific mutations than matched tissue. This finding may help targeted therapy and drug development. Furthermore, CSF samples collected at different time points could represent snap-shot genome landscape only at the time of acquisition. Our finding may help diagnosis and monitoring progressive disease for patients with $\mathrm{MB}$. This research is still ongoing. Thus, prospective studies are needed to confirm if CSF-ctDNA detection as liquid biopsy could be used in routine clinical therapy for MB, and if CSF-ctDNA gene mutations could guide targeted therapy in recurrent childhood MB.

Received: 17 October 2020; Accepted: 22 February 2021

Published online: 11 March 2021

\section{References}

1. Fiaschetti, G. et al. NOTCH ligands JAG1 and JAG2 as critical pro-survival factors in childhood medulloblastoma. Acta Neuropathol. Commun. 2, 39 (2014).

2. Louis, D. N. et al. The 2016 World Health Organization classification of tumors of the central nervous system: A summary. Acta Neuropathol. 131, 803-820 (2016).

3. Cefalo, G. et al. Temozolomide is an active agent in children with recurrent medulloblastoma/primitive neuroectodermal tumor: An Italian multi-institutional phase II trial. Neuro Oncol. 16, 748-753 (2014).

4. Bettegowda, C. et al. Detection of circulating tumor DNA in early- and late-stage human malignancies. Sci. Transl. Med. 6, $224 \mathrm{ra} 224$ (2014).

5. Mattox, A. K., Yan, H. \& Bettegowda, C. The potential of cerebrospinal fluid-based liquid biopsy approaches in CNS tumors. Neuro Oncol. 21, 1509-1518 (2019).

6. Snyder, M. W. et al. Cell-free DNA comprises an in vivo nucleosome footprint that informs its tissues-of-origin. Cell 164, 57-68 (2016).

7. Newman, A. M. et al. An ultrasensitive method for quantitating circulating tumor DNA with broad patient coverage. Nat. Med. 20, 548-554 (2014).

8. Mouliere, F. et al. Enhanced detection of circulating tumor DNA by fragment size analysis. Sci. Transl. Med. 10, eaat4921 (2018).

9. Wang, Y. et al. Detection of tumor-derived DNA in cerebrospinal fluid of patients with primary tumors of the brain and spinal cord. Proc. Natl. Acad. Sci. 112, 9704-9709 (2015).

10. Wu, X. et al. Circulating tumor DNA as an emerging liquid biopsy biomarker for early diagnosis and therapeutic monitoring in hepatocellular carcinoma. Int. J. Biol. Sci. 16, 1551-1562 (2020).

11. Li, H. \& Durbin, R. Fast and accurate short read alignment with Burrows-Wheeler transform. Bioinformatics 25, 1754-1760 (2009).

12. DePristo, M. A. et al. A framework for variation discovery and genotyping using next-generation DNA sequencing data. Nat. Genet. 43, 491-498 (2011).

13. Koboldt, D. C. et al. VarScan 2: Somatic mutation and copy number alteration discovery in cancer by exome sequencing. Genome Res. 22, 568-576 (2012).

14. Stewart, D. J. A critique of the role of the blood-brain barrier in the chemotherapy of human brain tumors. J. Neurooncol. 20, 121-139 (1994).

15. Van Vulpen, M., Kal, H. B., Taphoorn, M. J. \& El-Sharouni, S. Y. Changes in blood-brain barrier permeability induced by radiotherapy: Implications for timing of chemotherapy? (Review). Oncol. Rep. 9, 683-688 (2002). 
16. Hall, W. A. et al. Osmotic blood-brain barrier disruption chemotherapy for diffuse pontine gliomas. J. Neurooncol. 77, 279-284 (2006).

17. Fauquette, W., Amourette, C., Dehouck, M. P. \& Diserbo, M. Radiation-induced blood-brain barrier damages: an in vitro study. Brain Res. 1433, 114-126 (2012).

18. Skowron, P., Ramaswamy, V. \& Taylor, M. D. Genetic and molecular alterations across medulloblastoma subgroups. J. Mol. Med. (Berl.) 93, 1075-1084 (2015).

19. Northcott, P. A. et al. Subgroup-specific structural variation across 1000 medulloblastoma genomes. Nature 488, 49-56 (2012).

20. Woodworth, G. F. et al. Histopathological correlates with survival in reoperated glioblastomas. J. Neurooncol. 113, 485-493 (2013).

21. Momtaz, P. et al. Quantification of tumor-derived cell free DNA(cfDNA) by digital PCR (DigPCR) in cerebrospinal fluid of patients with BRAFV600 mutated malignancies. Oncotarget 7, 85430-85436 (2016).

22. Pan, C. et al. Molecular profiling of tumors of the brainstem by sequencing of CSF-derived circulating tumor DNA. Acta Neuropathol. 137, 297-306 (2019).

23. Robinson, G. et al. Novel mutations target distinct subgroups of medulloblastoma. Nature 488, 43-48 (2012)

24. Shi, X. et al. Modulation of p53 function by SET8-mediated methylation at lysine 382. Mol. Cell 27, 636-646 (2007).

25. Engelender, S. et al. Synphilin-1 associates with alpha-synuclein and promotes the formation of cytosolic inclusions. Nat. Genet. 22, 110-114 (1999).

26. Chung, K. K. et al. Parkin ubiquitinates the alpha-synuclein-interacting protein, synphilin-1: Implications for Lewy-body formation in Parkinson disease. Nat. Med. 7, 1144-1150 (2001).

27. Johnson, B. E. et al. Mutational analysis reveals the origin and therapy-driven evolution of recurrent glioma. Science 343, 189-193 (2014).

28. Kim, H. et al. Whole-genome and multisector exome sequencing of primary and post-treatment glioblastoma reveals patterns of tumor evolution. Genome Res. 25, 316-327 (2015).

29. Kim, J. et al. Spatiotemporal evolution of the primary glioblastoma genome. Cancer Cell 28, 318-328 (2015).

30. Wang, J. et al. Clonal evolution of glioblastoma under therapy. Nat. Genet. 48, 768-776 (2016).

31. Aihara, K. et al. Genetic and epigenetic stability of oligodendrogliomas at recurrence. Acta Neuropathol. Commun. 5, 18 (2017).

32. Miller, A. M. et al. Tracking tumour evolution in glioma through liquid biopsies of cerebrospinal fluid. Nature 565, 654-658 (2019).

\section{Acknowledgements}

We thank the patients and their families for participation in this study. We also thank Dr. Qian Chen and Dr. Guojun Li for assistance in mutation analysis; We thank members of the ThorGene (Beijing) Medical Technology Co., Ltd. for the bioinformatics analysis of DNA mutations.

\section{Author contributions}

Y.S. designed the work and wrote the main manuscript text, M.L., S.R. analyzed the data and prepared Tables 1, 2, 3 and 4 and Fig. 1, Y.L., J.Z., S.L., W.G., X.G., Y.W., collected samples and prepared data, J.L. done word processing, S.D. and W.W. supervised the study and helped in obtaining the funding, L.S., and Y.T. revised the manuscript. All the authors read and approved the final manuscript.

\section{Funding}

This work was supported by Special Fund of Pediatric Medical Coordinated Development Center of Beijing Hospitals Authority (Grant No. XTYB201816).

\section{Competing interests}

The authors declare no competing interests.

\section{Additional information}

Supplementary Information The online version contains supplementary material available at https://doi. org/10.1038/s41598-021-85178-6.

Correspondence and requests for materials should be addressed to W.W. or Y.T.

Reprints and permissions information is available at www.nature.com/reprints.

Publisher's note Springer Nature remains neutral with regard to jurisdictional claims in published maps and institutional affiliations.

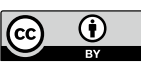

Open Access This article is licensed under a Creative Commons Attribution 4.0 International License, which permits use, sharing, adaptation, distribution and reproduction in any medium or format, as long as you give appropriate credit to the original author(s) and the source, provide a link to the Creative Commons licence, and indicate if changes were made. The images or other third party material in this article are included in the article's Creative Commons licence, unless indicated otherwise in a credit line to the material. If material is not included in the article's Creative Commons licence and your intended use is not permitted by statutory regulation or exceeds the permitted use, you will need to obtain permission directly from the copyright holder. To view a copy of this licence, visit http://creativecommons.org/licenses/by/4.0/.

(C) The Author(s) 2021 